\title{
Fast Detection Algorithm for Voltage Sags and Swells Based on Delta Square Operation for a Single-Phase Inverter System
}

\author{
Woo-Cheol Lee ${ }^{\dagger}$, Kook-Nam Sung* and Taeck-Kie Lee**
}

\begin{abstract}
In this paper, a new sag and peak voltage detector is proposed for a single-phase inverter using delta square operation. The conventional sag detector is from a single-phase digital phaselocked loop (DPLL) that is based on $\mathrm{d}-\mathrm{q}$ transformations using an all-pass filter (APF). The $\mathrm{d}-\mathrm{q}$ transformation is typically used in the three-phase coordinate system. The APF generates a virtual qaxis voltage component with a $90^{\circ}$ phase delay, but this virtual phase cannot reflect a sudden change in the grid voltage at the instant the voltage sag occurs. As a result, the peak value is drastically distorted, and it settles down slowly. A modified APF generates the virtual q-axis voltage component from the difference between the current and the previous values of the d-axis voltage component in the stationary reference frame. However, the modified APF cannot detect the voltage sag and peak value when the sag occurs around the zero crossing points such as $0^{\circ}$ and $180^{\circ}$, because the difference voltage is not sufficient to detect the voltage sag. The proposed algorithm detects the sag voltage through all regions including the zero crossing voltage. Moreover, the exact voltage drop can be acquired by calculating the q-axis component that is proportional to the d-axis component. To verify the feasibility of the proposed system, the conventional and proposed methods are compared using simulations and experimental results.
\end{abstract}

Keywords: Sag and Peak Detector, All-Pass Filter (APF), Digital Phase-Locked Loop (DPLL)

\section{Introduction}

Precise voltage control and voltage stability have gained significant importance in critical operational processes required in industries. Sensitive loads are significantly affected by power quality disturbances in the system, and voltage sags are among the most frequently occurring power quality problems. Voltage sags are defined as a decrease in the root mean square (rms) voltage below 0.9 $\mathrm{pu}$ of the nominal voltage at the power frequency for durations from 0.5 cycle to $1 \mathrm{~min}$ [1]. It is important that the system detects the phase and amplitude of the grid voltage when a problems occur in the grid; for this reason, a sag voltage detector has been previously proposed [2-4].

Most of the conventional peak detector methods have a time delay until detection. The RMS method has a time delay of 2-9 ms [5], the hybrid KF-RMS method has a time delay of 0.5-4 ms [6], and DVR has a 2 ms time delay [7]. Other methods report time delays ranging from 1-4 ms, with the maximum delay being generated at $0^{\circ}$ or $180^{\circ}$ for all methods. Recently, the fast peak detector has decreased

$\dagger \quad$ Corresponding Author: Dept. of Electrical, Electronic and Control Engineering, Hankyong National University, Korea.

(woocheol@hknu.ac.kr)

* R\&D Institute, GreenPower Company, Korea

(knsung@egreenpower.com)

** Dept. of Electrical, Electronic and Control Engineering, Hankyong

National University, Korea. (tklee@hknu.ac.kr)

Received: March 25, 2015; Accepted: October 26, 2015 the average detection delay to within $0.5 \mathrm{~ms}$ for any phase, and a sampling time of 3-5 cycles is needed [8]. Another conventional method is based on the d-q transformation using an all-pass filter (APF). The APF generates a virtual phase with a $90^{\circ}$ phase delay, but the virtual phase cannot reflect sudden changes in the grid voltage at the instant the voltage sag occurs. As a result, the peak value is significantly distorted and settles down slowly. Recently, a new sag detection method was implemented using the difference between the current and previous values of the grid voltage [8]. However, the disadvantage of this method is that it takes time to detect the sag voltage, and sometimes it cannot detect the voltage sag around the zero crossing. To solve these problems a new sag detector is proposed in this paper. There are two difference voltages. One difference voltage is the difference in values between the present and previous values of the d-axis voltage component, and the other difference voltage is the difference in values between the present difference value and the next difference value. The proposed detection method, called delta square operation, is implemented by using the difference voltages that exist because of these two difference voltages. The proposed method decreases the delay in the average detection time within one sampling time in any phase.

This paper consists of the following three sections: the system configuration (APF and digital phase-locked loop (DPLL); conventional detection method; and proposed detection method, simulations, and experimental results. 


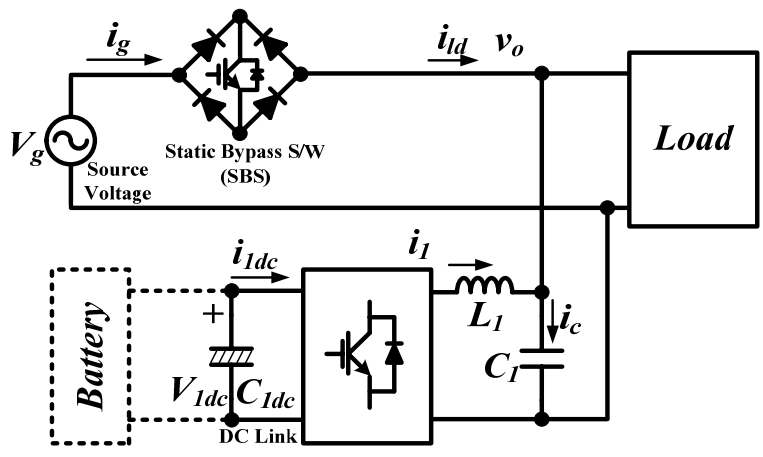

Fig. 1. Instantaneous voltage compensation system.

\section{System Configuration}

Fig. 1 shows the configuration of the instantaneous voltage compensation system [9]. When the grid voltage is within the normal range and the static bypass switch is turned on, then it operates in active power filter mode. When the grid voltage is beyond the normal range and the static bypass switch is turned off, then it operates in uninterruptible power supply (UPS) mode. To achieve seamless mode transfer, the static bypass switch, which consists of an insulated-gate bipolar transistor (IGBT), and a diode is used. However, if the grid voltage is not detected as soon as there is a voltage sag/swell, seamless mode transfer is not possible. Therefore, a new detection scheme that can detect the voltage drop is proposed.

\subsection{Conventional Sag Detector and DPLL}

Fig. 2 shows a conventional sag detector and the block diagram of a DPLL $[10,11] . V_{g d}^{s}$ is the d-axis component with the same magnitude and reverse phase as the that of the grid voltage, and $V_{g q}^{s}$ is the virtual q-axis component, which is generated using the APF from the grid voltage, The virtual phase $V_{\mathrm{g} q}^{s}$ can be obtained from the measured grid voltage $V_{g d}^{s}=-V_{g}$ by using the APF in the discrete-

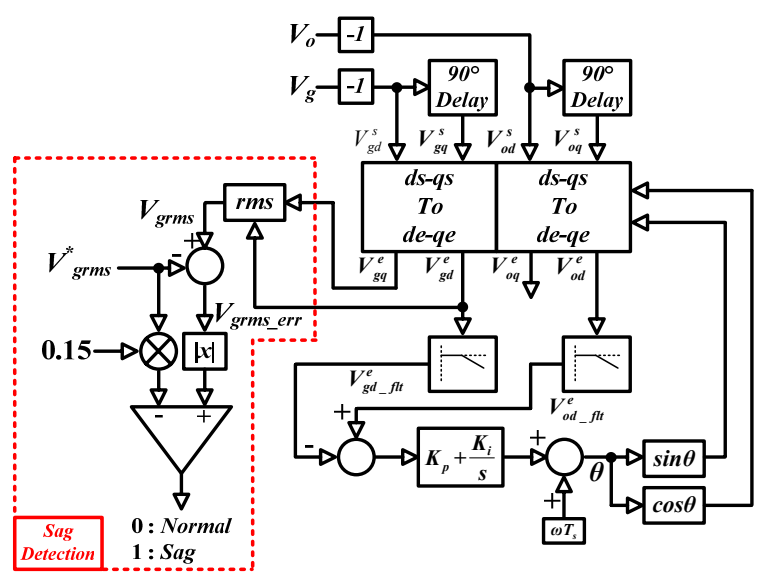

Fig. 2. Conventional sag detector and digital phase-lockedloop. time domain as follows:

$$
\begin{gathered}
V_{g d}^{s}(t)=-V_{g}(t) \\
V_{g q}^{s}(t)=-k V_{g q}^{s}(t-1)+k V_{g d}^{s}(t)+V_{g d}^{s}(t-1)
\end{gathered}
$$

where $k=\frac{T_{\text {samp }} \cdot \omega-2}{T_{\text {samp }} \cdot \omega+2}$

By using a synchronous rotating reference frame, the rms value of the grid voltage is obtained as follows:

$$
\begin{aligned}
& V_{g r m s}=\sqrt{\frac{\left(V_{g d}^{e}\right)^{2}+\left(V_{g q}^{e}\right)^{2}}{2}} \\
& V_{g r m s \_e r r}=V_{g r m s}-V_{g r m s}^{*}
\end{aligned}
$$

If Vgrms_err is beyond $15 \%$ of the absolute source voltage (reference, $0.15 \times V^{*}$ grms), then the source voltage sag is detected. However, there is a delay in detecting the sag because of the non-ideal phenomenon of the APF.

Fig. 3 shows the APF characteristics when the sag occurs in an entire section. Fig. 3 (a) shows that the APF generates a proper virtual phase when the sag occurs between $0-90^{\circ}$ and between $180-270^{\circ}$. In other words, if the polarities of the grid voltage and the virtual voltage are different, then the APF generates the proper virtual phase. However, when the sag occurs between 90-180 and between $270-360^{\circ}$, the APF generates the incorrect virtual

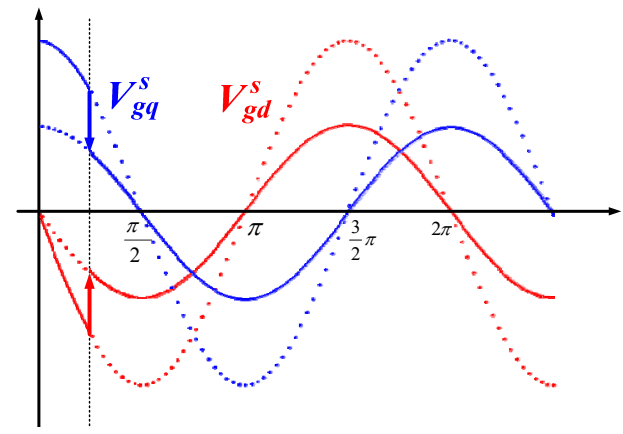

(a)

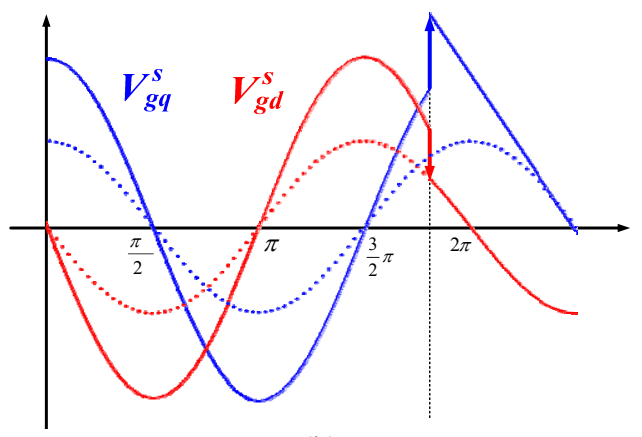

(b)

Fig. 3. APF characteristics: (a) Normal condition of APF $\left(0-90^{\circ}, 180-270^{\circ}\right)$; (b) Abnormal condition of APF $\left(90-180^{\circ}, 270-360^{\circ}\right)$. 
phase that is in the opposite direction to the decreased grid voltage. Moreover, if the polarities of the grid voltage and the virtual voltage are the same, the APF operates poorly. The APF output variation in the opposite direction is reflected in the DPLL operation, and thus the phase tracking time and peak voltage detection time are slowed down because of the unexpected disturbances to the DPLL.

\subsection{Conventional modified APF}

The modified APF is a technique used to check the grid voltage variations. The d-axis voltage component is generated from the current and previous values of the grid voltage, and the virtual q-axis component is generated using the same method.

Fig. 4 shows a conventional sag detection scheme using the difference between the current and previous values of the voltage.

$V_{g d}^{s}$ is the d-axis component with the same magnitude and reverse phase as that of the grid voltage. $V_{g q}^{s}$ is the virtual q-axis component, which is generated by using APF from grid voltage.

$$
\begin{aligned}
& \Delta V_{g d}^{s}=V_{g d}^{s}(t)-V_{g d}^{s}(t-1) \\
& =-A \sqrt{2} \cos (\omega t-\alpha) \cong-A \sqrt{2} \cos \omega t \\
& \Delta V_{g q}^{s}=V_{g q}^{s}(t)-V_{g q}^{s}(t-1) \\
& =-A \sqrt{2} \sin (\omega t-\alpha) \cong-A \sqrt{2} \sin \omega t
\end{aligned}
$$

where $\alpha=2 \pi f \cdot T_{\text {samp }} \cdot 0.5, A=V_{g} \cdot \sin \alpha \cdot 2$

$V_{g d}^{s}(t-1), V_{g q}^{s}(t-1)$ are the values for one step ahead voltage. $\Delta V_{g d}^{s}$ is equal to $-A \sqrt{2} \cos \omega t$, and the magnitude is much smaller than the real grid voltage. The peak value of the grid voltage, $\Delta V_{\text {gdq }}^{s}$ peak , is calculated under normal conditions, and is defined as follows:

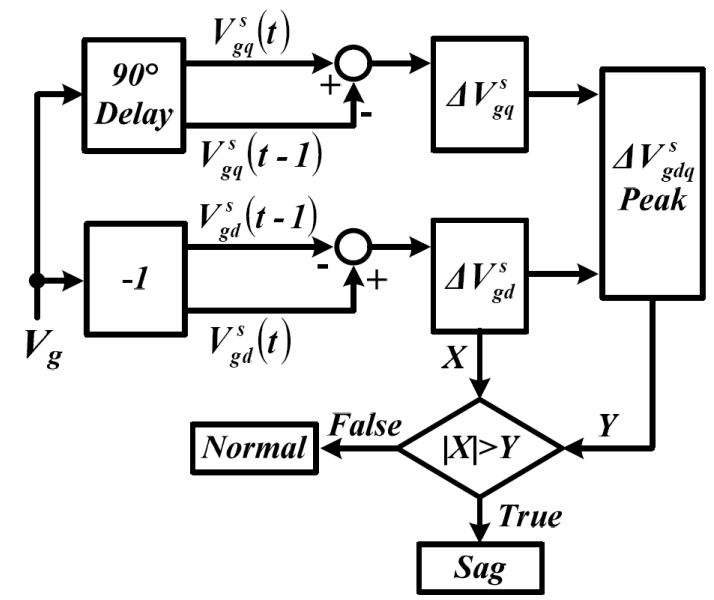

Fig. 4. Conventional sag detection scheme using the difference between current and previous values of the voltage.

$$
\Delta V_{g d q_{-} p e a k}^{s}=\sqrt{\left(\Delta V_{g d}^{s}\right)^{2}+\left(\Delta V_{g q}^{s}\right)^{2}}=A \sqrt{2}
$$

$\Delta V_{g d}^{s}$ is then compared with $\Delta V_{g d q_{-} p e a k}^{s}$, and if $\left|\Delta V_{g d}^{s}\right|>\Delta V_{g d q_{-} \text {peak }}^{s}$, then it means that a sag has occurred.

However, as shown in Fig. 5 and Table 1, when the sag occurs at around zero voltage, it cannot be detected because the variation in the voltage is small owing to the characteristic of the sinusoidal waveform. The parameters of y-axis in Fig. 5 indicate the amplitude of the sine function.

For example, at $10 \%$ of the sag voltage when the sampling time is $100 \mu \mathrm{s}$, sags within $\pm 22.1^{\circ}$ were not detected, and at $50 \%$ of the sag voltage when the sampling time is $100 \mu \mathrm{s}$, sags within $\pm 4.3^{\circ}$ were not detected. If the sampling time is increased to $200 \mu \mathrm{s}$, the length of time needed before detecting the sag voltage increases further.

\subsection{Proposed sag / Peak detection method}

Fig. 6 shows the block diagram for the proposed sag

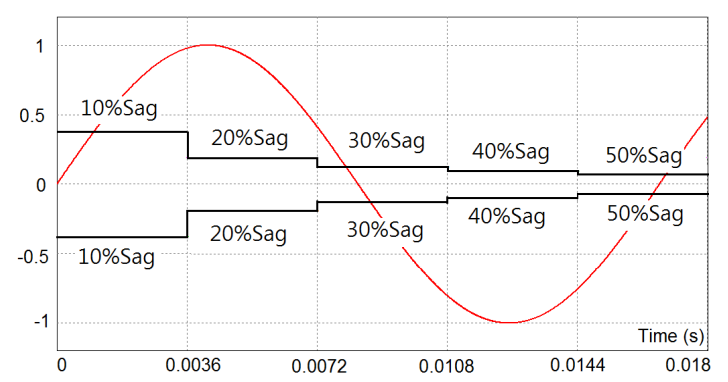

(a)

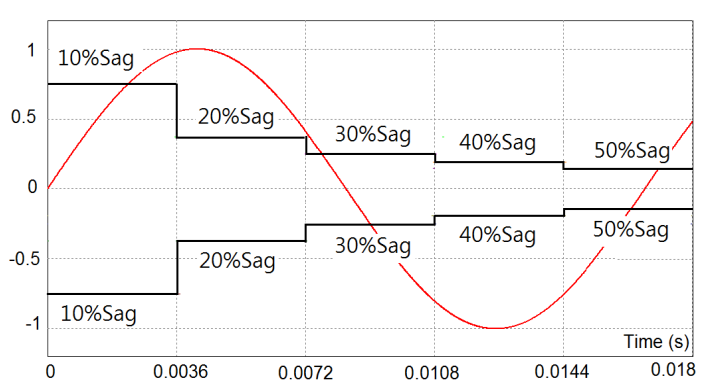

(b)

Fig. 5. Sag detection range of the source voltage depending on the voltage sag: (a) When the sampling time is $100 \mu \mathrm{s}$; (b) When the sampling time is $200 \mu \mathrm{s}$.

Table 1. System parameters

\begin{tabular}{c|c|c}
\hline Voltage sag & Tsamp $=100 \mu \mathrm{s}$ & Tsamp $=200 \mu \mathrm{s}$ \\
\hline $10 \% \mathrm{Sag}$ & $\pm 22.1^{\circ}(1.03 \mathrm{~ms})$ & $\pm 48.9^{\circ}(2.27 \mathrm{~ms})$ \\
\hline $20 \% \mathrm{Sag}$ & $\pm 10.9^{\circ}(503 \mu \mathrm{s})$ & $\pm 21.2^{\circ}(1.03 \mathrm{~ms})$ \\
\hline $30 \% \mathrm{Sag}$ & $\pm 7.2^{\circ}(334 \mu \mathrm{s})$ & $\pm 14.6^{\circ}(674 \mu \mathrm{s})$ \\
\hline $40 \% \mathrm{Sag}$ & $\pm 5.4^{\circ}(251 \mu \mathrm{s})$ & $\pm 10.9^{\circ}(503 \mu \mathrm{s})$ \\
\hline $50 \% \mathrm{Sag}$ & $\pm 4.3^{\circ}(200 \mu \mathrm{s})$ & $\pm 8.7^{\circ}(402 \mu \mathrm{s})$ \\
\hline
\end{tabular}




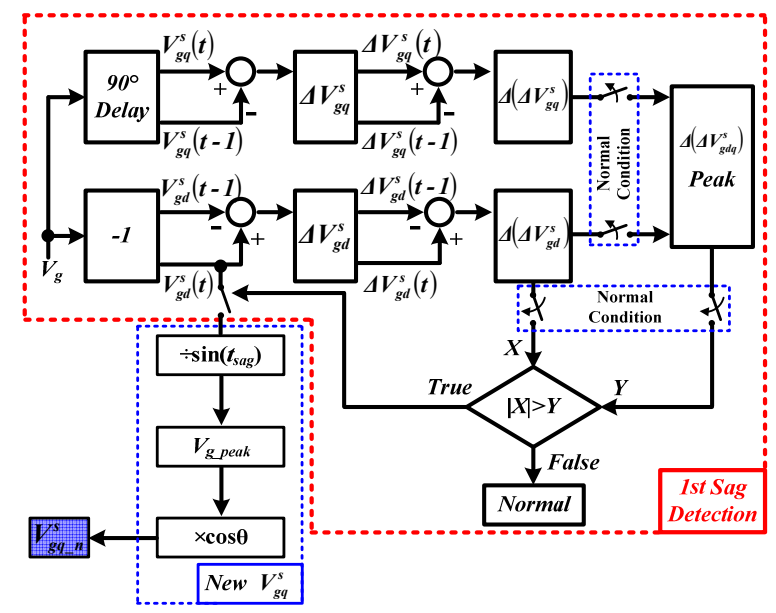

Fig. 6. Proposed detection of $1^{\text {st }}$ sag / new- $V_{g q}^{s}$.

detection, and the virtual new q-axis voltage, $V_{g d}^{s}{ }_{n}$, can be acquired using the proposed method.

The $1^{\text {st }}$ component of the difference voltages is generated through $\Delta V_{g d}^{s}$ and $\Delta V_{g q}^{s}$ that are based on the value of the present/one step ahead $V_{g d}^{s}$ and $V_{g q}^{s}$, respectively. Similar to Eqs. (5) and (6), the $2^{\text {nd }}$ component of the difference voltage is acquired as follows.

$$
\begin{aligned}
& \Delta\left(\Delta V_{g d}^{s}(t)\right)=\Delta V_{g d}^{s}(t)-\Delta V_{g d}^{s}(t-1) \\
& =B \sqrt{2} \sin (\omega t-\alpha) \cong B \sqrt{2} \sin \omega t \\
& \Delta\left(\Delta V_{g q}^{s}(t)\right)=\Delta V_{g q}^{s}(t)-\Delta V_{g q}^{s}(t-1) \\
& =-B \sqrt{2} \cos (\omega t-\alpha) \cong-B \sqrt{2} \cos \omega t
\end{aligned}
$$

where $\alpha=2 \pi f \cdot T_{\text {samp }} \cdot 0.5, B=A \cdot 2 \alpha$

The peak value of the grid voltage $-\left(\Delta V_{g d q}^{s}\right)_{\text {peak }}$, is calculated under normal conditions. The peak value can be found, as follows:

$$
\Delta\left(\Delta V_{g d q}^{s}\right)_{\text {peak }}=\sqrt{\left\{\Delta\left(\Delta V_{g d}^{s}\right)\right\}^{2}+\left\{\Delta\left(\Delta V_{g q}^{s}\right)\right\}^{2}}=B \sqrt{2}
$$

$\Delta\left(\Delta V_{g d q}^{s}\right)_{\text {peak }}$ is the peak value of the grid voltage, and is updated every hour under normal conditions. By comparing $\Delta\left(\Delta V_{g d q}^{s}\right)_{\text {peak }}$ with $\Delta\left(\Delta V_{g d}^{s}\right)$, if $\Delta\left(\Delta V_{g d}^{s}\right)$ existed within $\pm \Delta\left(\Delta V_{g d q}^{s}\right)_{\text {peak }}$, then the grid voltage is normal, but if $\Delta\left(\Delta V_{g d}^{s}\right)$ becomes greater than $\pm \Delta\left(\Delta V_{g d q}^{s}\right)_{\text {peak }}$, then there is a failure. After recognizing the failure, the system calculates the peak value of the grid voltage, as shown in Eq. (11). In other words, when the sag occurs, the peak voltage of the grid can be calculated by using the grid voltage and phase. The virtual phase $V_{g q_{-} n}^{s}$ can be obtained by using the peak voltage of the grid and the virtual phase.

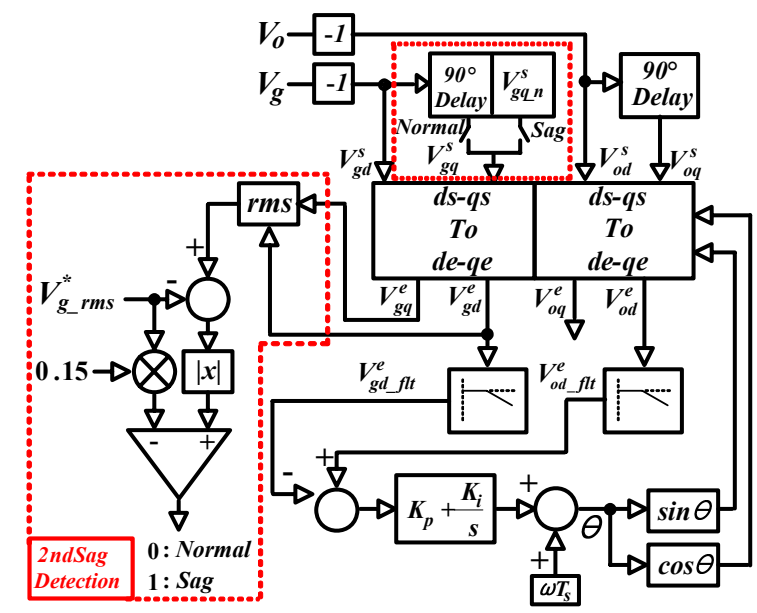

Fig. 7. Proposed sag/peak detection using the new $V_{g q_{-} n}^{s}$.

$$
V_{g_{-} \text {peak }}=\left|\frac{V_{g d}^{s}\left(t_{\text {sag }}\right)}{\sin \left(t_{\text {sag }}\right)}\right|=\left|\frac{-V_{m} \sin \left(t_{\text {sag }}\right)}{\sin \left(t_{\text {sag }}\right)}\right|
$$

The virtual phase $V_{g q_{-} n}^{s}$ can be obtained from the measured grid voltage by using Eq. (12).

$$
V_{g q}^{s}\left(t_{\text {sag }}\right)=V_{g_{-} \text {peak }} \times \cos \left(\theta_{\text {sag }}\right)=V_{g q_{-} n}^{s}
$$

$V_{g q}^{s}\left(t_{s a g}\right)$ is the same as the new $V_{g q_{-} n}^{s}$, as shown in Eq. (5). As shown in Fig. 7, the new peak value is created through the output of the rotating coordinate system using the new- $V_{g q_{n} n}^{s}$. The system detects the sag immediately because of this peak value. The APF generates a proper virtual phase when the sag occurs between $0-90^{\circ}$ and between $180-270^{\circ}$, but when the sag occurs between 90 $180^{\circ}$ and between $270-360^{\circ}$, the APF generates the incorrect virtual phase. However, when the proposed method is used, the problem of the APF can be solved.

Assume that a fixed percentage, $x$, of the voltage sag occurred at time $(t+1)$. Then the following equations apply:

$$
\begin{aligned}
& V_{g d}^{s}(t-1)=-\sqrt{2} V \sin (\omega t-2 \alpha) \\
& V_{g d}^{s}(t)=-\sqrt{2} V \sin \omega t \\
& V_{g d}^{s}(t+1)=-\sqrt{2} V(1-x) \sin (\omega t+2 \alpha)
\end{aligned}
$$

Based on the value of the present/one step ahead $V_{g d}^{s}$ and $V_{g q}^{s}$, the $1^{\text {st }}$ component of the difference voltage was generated through $\Delta V_{g d}^{s}$ and $\Delta V_{g q}^{s}$.

$$
\begin{gathered}
\Delta V_{g d}^{s}(t)=V_{g d}^{s}(t)-V_{g d}^{s}(t-1) \cong-A \sqrt{2} \cos \omega t \\
\Delta V_{g d}^{s}(t+1)=V_{g d}^{s}(t+1)-V_{g d}^{s}(t) \\
\cong-\sqrt{2} V\{-x \sin \omega t+2 \alpha(1-x) \cos \omega t\}
\end{gathered}
$$

The difference voltage of the $2^{\text {nd }}$ component was 


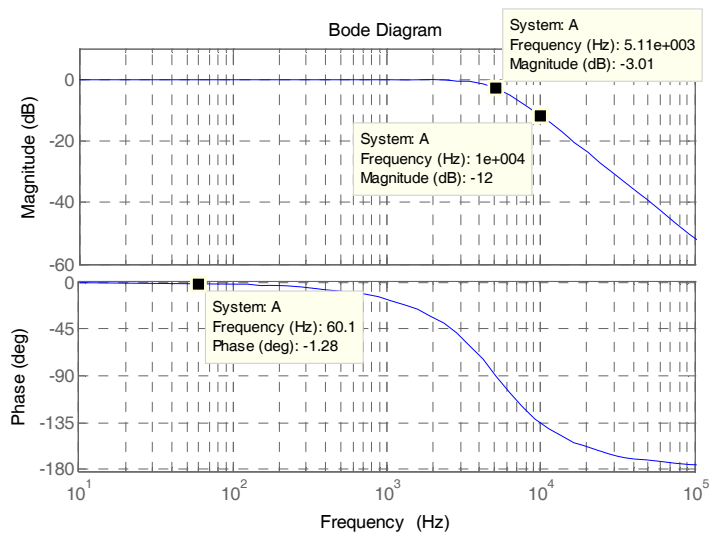

(a)
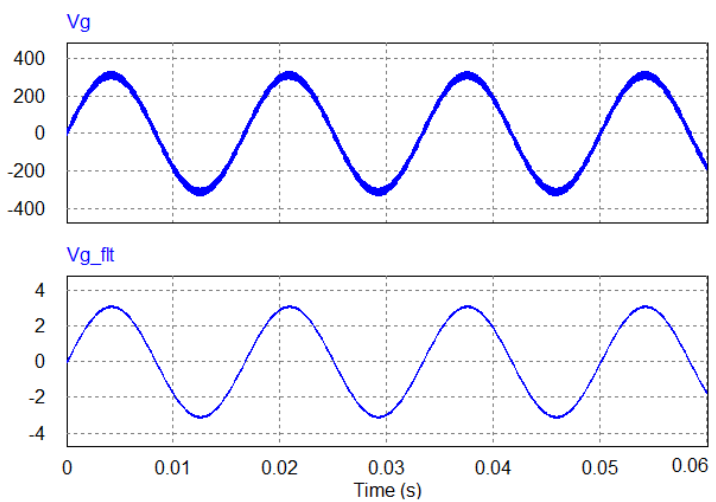

(b)

Fig. 8. Characteristics of the low-pass filter: (a) Frequency domain; (b) Time domain.

generated by $\Delta V_{g d}^{s}(t+1)$ and $\Delta V_{g d}^{s}(t)$.

$$
\begin{aligned}
& \Delta\left(\Delta V_{g d}^{s}(t+1)\right)=\Delta V_{g d}^{s}(t+1)-\Delta V_{g d}^{s}(t) \\
& =\sqrt{2} V \cdot x \cdot(\sin \omega t+2 \alpha \cos \omega t)
\end{aligned}
$$

The minimum voltage drop which can be detected at $\mathrm{t}=$ 0 is calculated as follows.

$$
\begin{aligned}
& \Delta\left(\Delta V_{g d}^{s}(1)\right)=\sqrt{2} V \cdot x \cdot(\sin 0+2 \alpha \cos 0) \\
&=\sqrt{2} V \cdot x \cdot 2 \alpha \\
& \sqrt{2} V \cdot 2 \alpha=B \sqrt{2} \\
& x=\frac{B \sqrt{2}}{\sqrt{2} \cdot V \cdot 2 \alpha}(\%)
\end{aligned}
$$

The minimum detection level of the sag voltage can be manipulated using $B$.

\subsection{Solution for the noise and harmonics on the grid side}

However, the proposed method has a disadvantage with respect to the noise and harmonics of the grid side, because when detecting the grid voltage, the difference in the difference voltage is used. Therefore, when detecting the grid voltage $-40 \mathrm{~dB} /$ decade, low-pass Butterworth filters used in controller implementation are realized with a cutoff frequency of $5.1 \mathrm{kHz}$. The phase delay of the filter is about $60 \mu$ s at the fundamental frequency.

\section{Simulation Results}

Simulations of the proposed algorithms were performed using the simulation tool PSIM (Powersim Inc.), and the system parameters used in the experiment and simulations are listed in Table 2 . The sag voltage dropped to $50 \%$ of its nominal value.

Fig. 9 shows the characteristics of the APF when the sag/swell occurs at a specific phase. As shown in Fig. 9(a), the APF generates a proper virtual phase when the

Table 2. System parameters

\begin{tabular}{c|c}
\hline Parameter & Value \\
\hline Source Voltage $(V g)$, Rating & $208 \mathrm{~V}, 60 \mathrm{~Hz}$ \\
\hline Sag Source Voltage $(V g)$ & $104 \mathrm{~V}, 60 \mathrm{~Hz}$ \\
\hline Switching Frequency & $10 \mathrm{kHz}$ \\
\hline
\end{tabular}

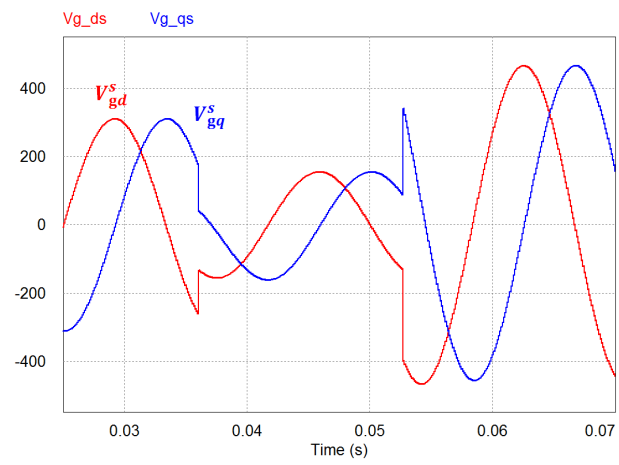

(a)

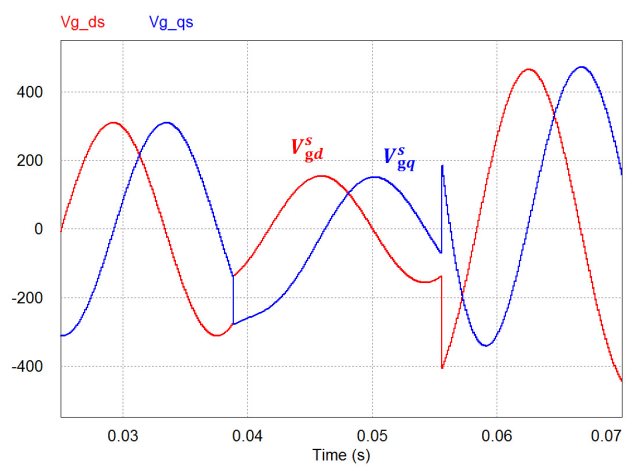

(b)

Fig. 9. 50\% sagged/swelled grid voltage for the conventional APF: (a) Sagged/swelled grid voltage at $60^{\circ}$; (b) Sagged $/$ swelled grid voltage at $120^{\circ}$. 


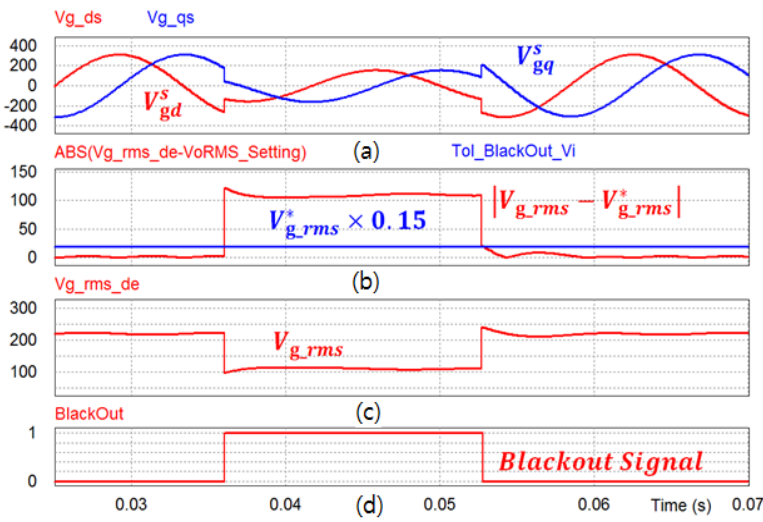

Fig. 10. 50\% sagged/returned grid voltage for the conventional method at $60^{\circ}$ : (a) Stationary reference frame $V_{\mathrm{g} d}^{s}$ and $V_{\mathrm{gq}}^{s}$; (b) Sag detection level; (c) Detected rms voltage $V_{\mathrm{g} \text { rms }}$; (d) Blackout signal (BS).

sag/swell occurs between $0^{\circ}$ and $90^{\circ}$ and between $180^{\circ}$ and $270^{\circ}$, respectively. In this case, the polarities of $V_{\mathrm{g} d}^{s}$ and $V_{\mathrm{g} q}^{s}$ are opposite to each other. However, Fig. 9(c) shows that the APF generates opposite virtual phases when the sag/swell occurs between $90^{\circ}$ and $180^{\circ}$ and between $270^{\circ}$ and $360^{\circ}$, respectively. In this case, the polarities of $V_{\mathrm{g} d}^{S}$ and $V_{\mathrm{g} q}^{s}$ are the same.

Fig. 10 shows the grid voltage obtained for the conventional method using the d-q transformation at $60^{\circ}$ when sagged by $50 \%$. Fig. 10(a) shows $V_{\mathrm{g} d}^{s}$ from the grid voltage and a $90^{\circ}$ phase-lagged virtual waveform $V_{\mathrm{g} q}^{s}$ generated by the APF. When $V_{\mathrm{g} d}^{s}$ sags at $60^{\circ}, V_{\mathrm{g} q}^{s}$ reflects the proper sag voltage value, as shown in Fig. 10 (a). In other words, when the grid voltage is decreased by $50 \%$ at $60^{\circ}$, the virtual phase $V_{\mathrm{g} q}^{s}$ is also decreased.

Fig. 10(b) shows the sag voltage detection level. The sag is detected when the source voltage drops to $15 \%$ of the nominal rms value. Fig. 10(c) shows the rms value of the grid voltage in Eqs. (3) and (4). Fig. 10(d) shows the detected blackout signal. Because of the proper virtual waveform of $V_{\mathrm{g} q}^{s}$, the sag voltage was detected properly.

Fig. 11 shows the grid voltage for the conventional method obtained using the d-q transformation at $310^{\circ}$ when sagged by $50 \%$. When $V_{\mathrm{g} d}^{s}$ sags at $310^{\circ}, V_{\mathrm{g} q}^{s}$ reflects the incorrect sag voltage value, as shown in Fig. 11(a). Namely, when the grid voltage is decreased by $50 \%$ at $310^{\circ}$, the virtual phase $V_{\mathrm{g} q}^{s}$ is increased. Therefore, it takes time to calculate the rms value of the grid voltage. As shown in Fig. 11(d), it takes $1.5 \mathrm{msec}$ to detect the sag. The blackout signal is represented by a zero (0) when the system has normal conditions, and if the system has abnormal conditions, the BS is represented by a one (1).

Fig. 12 shows the sag detection characteristic at $60^{\circ}$ when the difference voltage between the current and previous values is used.

The values of $V_{\mathrm{gq}_{-} \mathrm{n}}^{s}, \Delta V_{\mathrm{g} d}^{s}, \Delta V_{\mathrm{gdg} \_ \text {peak }}^{s}, \Delta\left(\Delta V_{\mathrm{g} d}^{s}\right)$, and

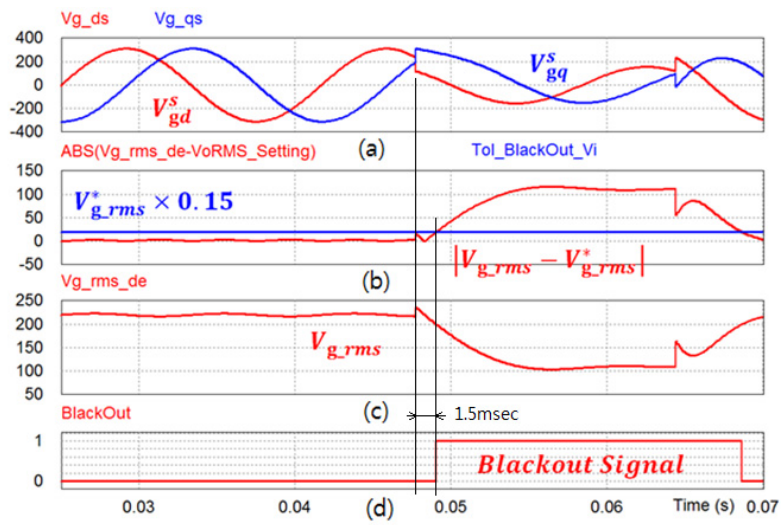

Fig. 11. 50\% sagged/returned grid voltage for the conventional method at $310^{\circ}$ : (a) Stationary reference frame $V_{\mathrm{g} d}^{s}$ and $V_{\mathrm{gq}}^{s}$; (b) Sag detection level; (c) Detected rms voltage $V_{\mathrm{g} \text { rms }}$; (d) Blackout signal.

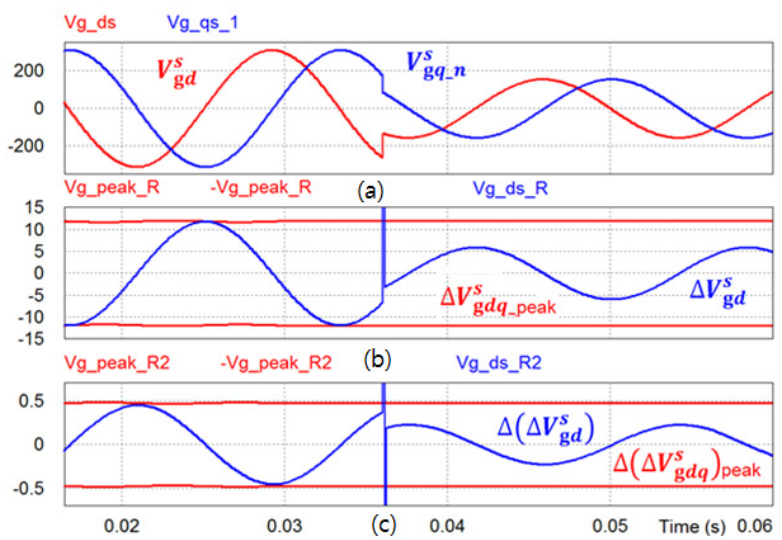

Fig. 12. Voltage sag detection characteristics around $60^{\circ}$ when the difference voltage between the current and previous values is used: (a) Stationary reference frame $V_{\mathrm{g} d}^{s}$ and $V_{\mathrm{gq} \_\mathrm{n}}^{s}$; (b) Difference voltage $\Delta V_{\mathrm{g} d}^{s}$ and peak voltage $\Delta V_{\mathrm{gdg} \text { peak }}^{S}$; (c) Difference voltage, $\Delta\left(\Delta V_{\mathrm{g} d}^{s}\right)$, and peak voltage $\Delta\left(\Delta V_{\mathrm{gdg}}^{s}\right)_{\text {peak }}$.

$\Delta\left(\Delta V_{\mathrm{g} d}^{s}\right)$ come from Eqs. (12), (5), (7), (8), and (10), respectively.

Fig. 12(a) shows the voltage sag detection characteristics when the difference voltage between the current and previous values of the d-axis voltage component is used in the stationary reference frame. The d-axis voltage component $\left(V_{\mathrm{g} d}^{s}\right)$ is the same as that of the grid voltage $\left(-V_{\mathrm{g}}\right)$. The value $\Delta V_{\mathrm{g} d}^{s}$ is compared with $\Delta V_{\mathrm{gdq} \_ \text {peak. If }}^{s}$ $\left|\Delta V_{\mathrm{g} d}^{s}\right|>\Delta V_{\mathrm{gdq} \_ \text {peak }}^{s}$, it indicates that the sag occurs as shown in Fig. 12(b). Fig. 12(c) shows the proposed method The result is almost the same as that obtained with the conventional method.

Fig. 13 shows the voltage sag detection characteristic at $0^{\circ}$. Fig. 13(b) shows that the voltage sag is not detected because the difference voltage between the current and 


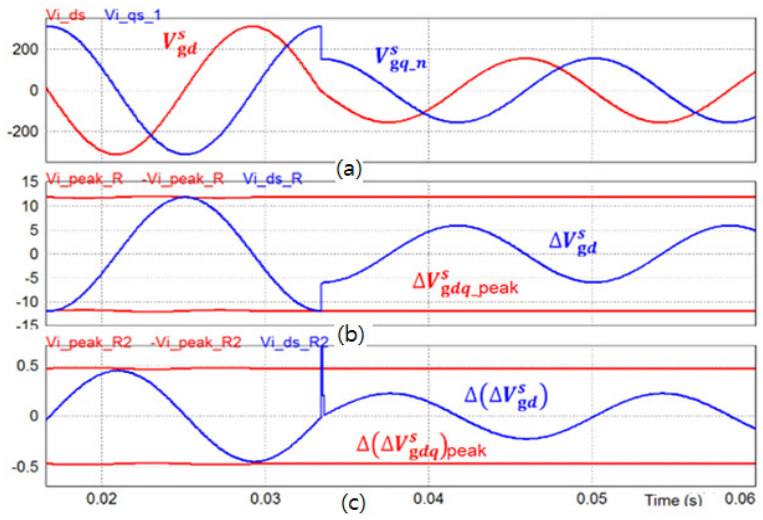

Fig. 13. Voltage sag detection characteristic at $0^{\circ}$ when the difference voltage between the current and previous values is used: (a) Stationary reference frame $V_{\mathrm{g} d}^{s}$ and $V_{\mathrm{gq} \mathrm{n}}^{s}$; (b) Difference voltage $\Delta V_{\mathrm{g} d}^{s}$ and peak voltage $\Delta V_{\text {gdg_peak }}^{s}$; (c) Difference voltage, $\Delta\left(\Delta V_{\mathrm{g} d}^{S}\right)$, and peak voltage $\Delta\left(\Delta V_{\mathrm{gdg}}^{S}\right)_{\text {peak }}$.

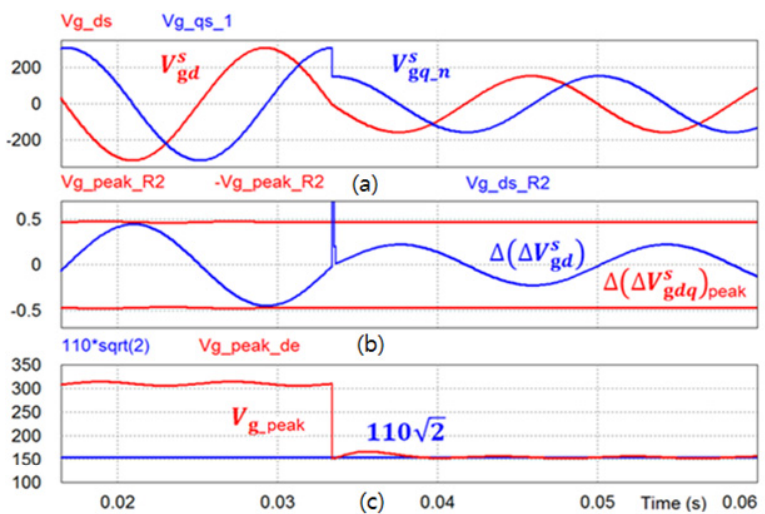

Fig. 14. 50\% sagged grid voltage at $0^{\circ}$ : (a) Stationary reference frame $V_{\mathrm{g} d}^{s}$ and $V_{\mathrm{gq} \_\mathrm{n}}^{s}$; (b) Difference voltage and $\Delta\left(\Delta V_{\mathrm{g} d}^{s}\right)$ and peak voltage $\Delta\left(\Delta V_{\text {gqd }}^{s}\right)_{\text {peak }} ;$ (c) Peak grid voltage.

previous values is not above the peak voltage level $\Delta V_{\text {gdg_peak }}^{s}$. This is because the source voltage is about zero, and the difference voltage is also too small. However, as shown in Fig. 13(c), the proposed method detects the voltage sag. Because of the voltage detection, the virtual qaxis voltage component is acquired immediately using Eq. (12).

The proposed sag/peak detection methods were shown in Fig. 14. The value of $V_{\text {g_peak }}$ is the peak of the grid voltage obtained using the synchronous d-q transformation. The sag was detected at $60^{\circ}$ and $0^{\circ}$. Because of the sag detection, the peak grid voltage was acquired immediately.

\section{Experimental Results}

In order to verify the proposed strategy, the algorithm

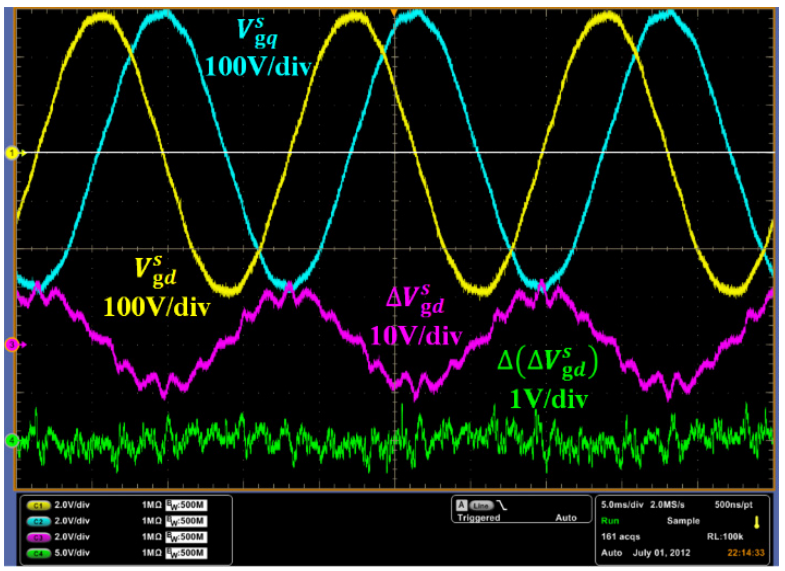

Fig. 15. Experimental results of the grid voltage. Ch 1. $V_{\mathrm{g} d}^{s}$ : grid voltage $(100 \mathrm{~V} / \mathrm{div}) . \mathrm{Ch} 2 . V_{\mathrm{gq}}^{s}$ : virtual source voltage $(100 \mathrm{~V} / \mathrm{div}) . \mathrm{Ch} 3 . \Delta V_{\mathrm{gd}}^{s}$ : conventional difference voltage $(10 \mathrm{~V} / \mathrm{div})$. Ch 4. $\Delta\left(\Delta V_{\mathrm{gd}}^{s}\right)$ : proposed difference voltage $(1 \mathrm{~V} / \mathrm{div})$.

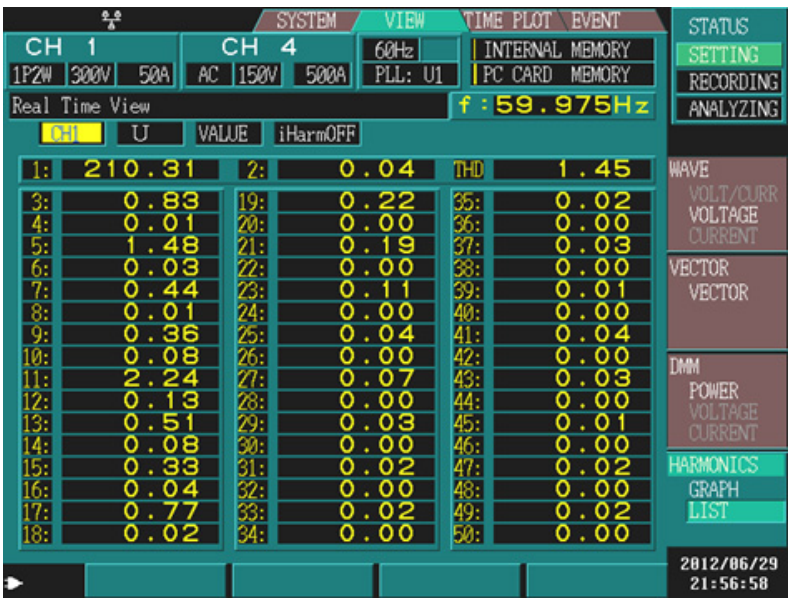

Fig. 16. Harmonic analysis of the grid voltage obtained using power analyzer.

was implemented using a digital signal processor (TMS 320C33). A single 32-bit floating-point DSP with a singlecycle execution time of 13.3 ns was used, and the switching period of the PWM was $100 \mu \mathrm{s}$. The system parameters are listed in Table 1. The nominal input voltage was 208 V. Voltage sags were simulated with a tap changing transformer and switching devices so that various voltage sag levels could be generated. In this study, the source voltage was decreased from 208-104 V during the sag event.

Fig. 15 shows the derived components obtained from the grid voltage. The waveforms of the d-axis voltage component $\left(V_{\mathrm{g} d}^{s}\right)$ and q-axis voltage component $\left(V_{\mathrm{gq}}^{s}\right)$ are slightly distorted, and the difference voltages $\left(\Delta V_{\mathrm{g} d}^{s}\right.$, $\left.\Delta\left(\Delta V_{\mathrm{g} d}^{S}\right)\right)$ are significantly distorted.

Fig. 16 shows the harmonic analysis results for the grid voltage; the results were obtained using a power analyzer. 


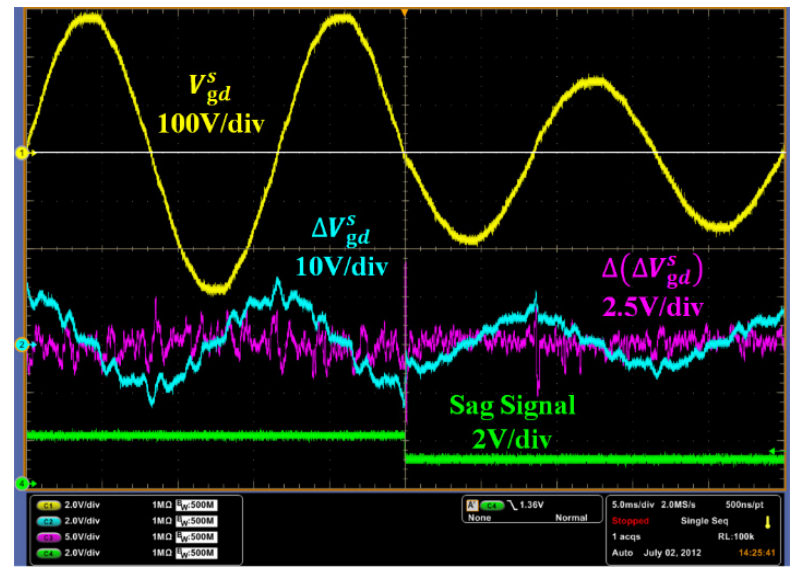

(a)

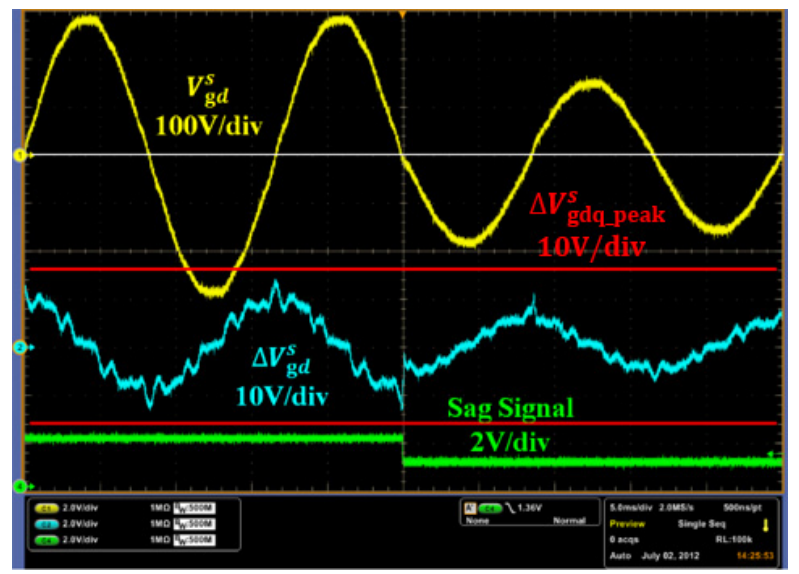

(b)

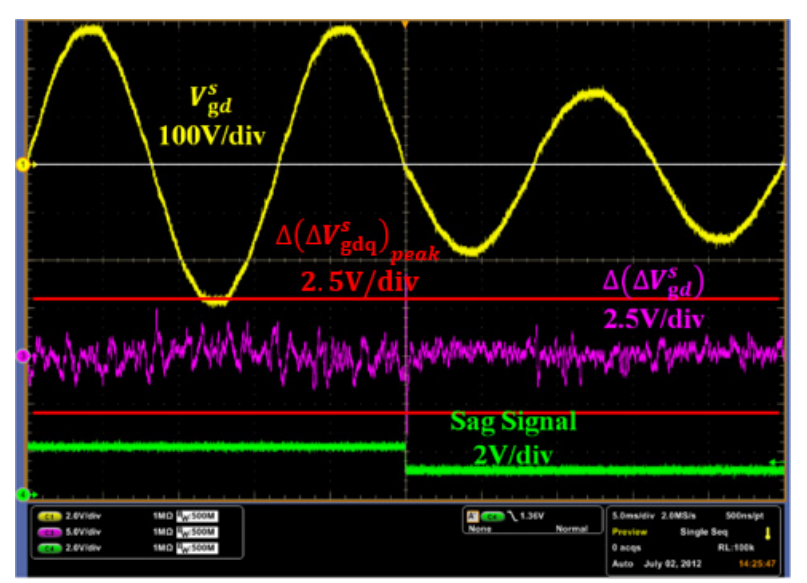

(c)

Fig. 17. Comparison of sag detection waveforms obtained for $V_{\mathrm{g} d}^{s}, \Delta\left(\Delta V_{\mathrm{g} d}^{s}\right)$. Ch 1: $V_{\mathrm{g} d}^{s}$ : grid voltage (100 $\mathrm{V} /$ div), Ch 2: $\Delta V_{\mathrm{g} d}^{s}$ : difference voltage (10 $\mathrm{V} /$ div) ), Ch 3: $\Delta\left(\Delta V_{\mathrm{g} d}^{s}\right):$ difference voltage (2.5 $\mathrm{V} /$ div). Ch 4: sag ordering signal $(2.5 \mathrm{~V} / \mathrm{div})$ : (a) Sag detection waveform obtained for $\Delta V_{\mathrm{g} d}^{s}$, $\Delta\left(\Delta V_{\mathrm{g} d}^{s}\right)$; (b) Sag detection waveform obtained for $\Delta V_{\mathrm{g} d}^{s}$; (c) Sag detection waveform obtained for $\Delta\left(\Delta V_{\mathrm{g} d}^{s}\right)$.

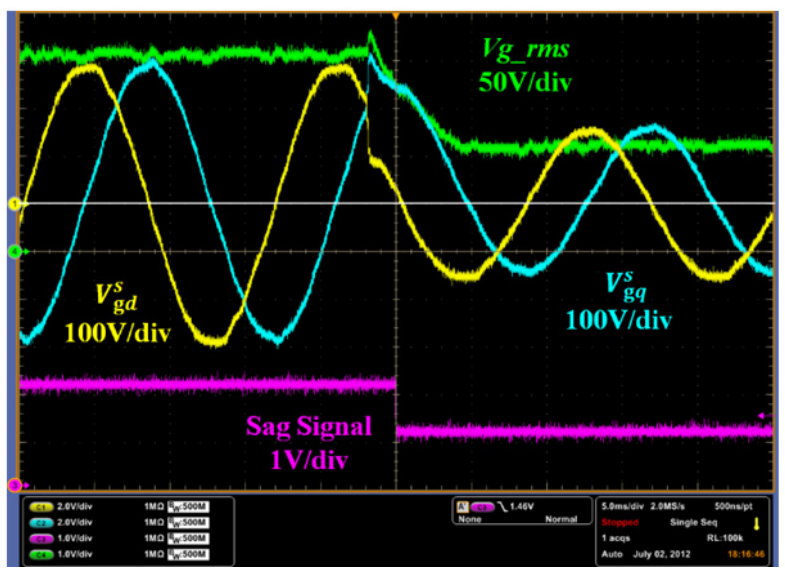

(a)

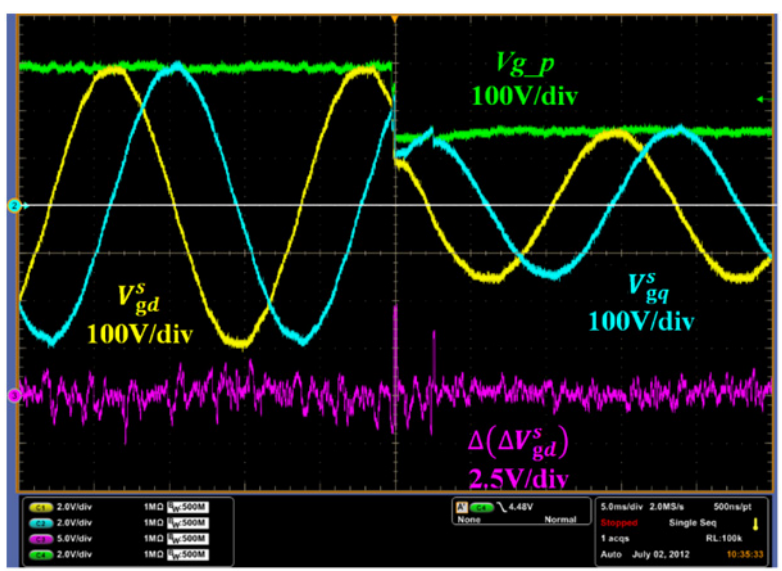

(b)

Fig. 18. Comparison of waveforms for sag detections: (a) Occurrence of a sag at $310^{\circ}$ using the conventional method; (b) Occurrence of a sag at $310^{\circ}$ using the proposed method.

\section{(Model: HIOKI 3194 Motor/Harmonic Hitesteor)}

The figure shows that the grid voltage includes the $3^{\text {rd }}$, $5^{\text {th }}$, and $7^{\text {th }}$ harmonic components Therefore, the difference voltages $\left(\Delta V_{\mathrm{g} d}^{s}, \Delta\left(\Delta V_{\mathrm{g} d}^{s}\right)\right)$ are significantly distorted.

Fig. 17 shows the sag detection waveform that was used to compare $\Delta V_{\mathrm{g} d}^{s}$ with $\Delta\left(\Delta V_{\mathrm{g} d}^{s}\right)$. The sag occurred at $0^{\circ}$. Fig. 17(b) and Fig. 17(c) were divided by Fig. 17(a). The occurrence of a sag can be detected if $\Delta V_{\mathrm{g} d}^{s}$ and $\Delta\left(\Delta V_{\mathrm{g} d}^{s}\right)$ matches each peak value. However, in the case of Fig. 17(b), the variation in $\Delta V_{\mathrm{g} d}^{s}$ was too small to detect the sag. Whereas, in the case of Fig. 17(c), the sag detection became possible because $\Delta\left(\Delta V_{\mathrm{g} d}^{s}\right)$ matched the peak value of $\Delta\left(\Delta V_{\mathrm{g} d}^{s}\right)$. The sag signal of $\mathrm{Ch} 4$ was output when the system recognized the sag. During normal conditions, this value maintains by $2 \mathrm{~V}$ and if there is a problem with the grid voltage, the sag signal changes by $1 \mathrm{~V}$.

Fig. 18 shows a comparison of the conventional and proposed methods. In this study, the grid voltage decreased from 208-104 V during the sag event. Fig. 18(a) shows the 
results for the conventional method. When $V_{\mathrm{g} d}^{s}$ sags at $310^{\circ}, V_{\mathrm{g} q}^{s}$ reflects the incorrect sag voltage value, as shown in Fig. 18(a). Namely, when the grid voltage is decreased by $50 \%$ at $310^{\circ}$, the virtual phase $V_{\mathrm{g} q}^{s}$ is increased. Therefore, it takes time to calculate the rms value of the grid voltage. However, Fig. 18(b) shows the proposed method, which shows that when the grid voltage is decreased by $50 \%$ at $310^{\circ}$, the sag is detected immediately, the virtual phase $V_{\mathrm{g} q}^{s}$ is decreased, and the rms value of the grid voltage is also calculated immediately.

\section{Conclusion}

In this paper, a new sag and peak voltage detector for a single-phase inverter was proposed. The conventional sag detector is from a single-phase DPLL that is based on d-q transformations using an APF. The APF generates a virtual q-axis voltage component with a $90^{\circ}$ phase delay, but this virtual phase cannot reflect sudden changes in the grid voltage at the instant when voltage sags between $0^{\circ}$ and $90^{\circ}$ and between $180^{\circ}$ and $270^{\circ}$. As a result, the peak value is significantly distorted, and settles down slowly. Moreover, the settling time of the peak value is too long.

To reduce these delays, the difference voltage between the current and previous values of the voltage component was used in the stationary reference frame, but the system could not recognize the sag around the zerocrossing point. Therefore, the proposed method used the difference voltage of the 2 nd component that was the voltage difference between the current value and of one step ahead value. The proposed algorithm could detect the sag voltage through all regions including the zero crossing voltage. Moreover, the exact voltage drop could be acquired by calculating the q-axis component, which is proportional to the d-axis component. The control algorithm and mathematical models were proposed, and simulation and experiment results were presented to verify the performance of the proposed control strategy. The proposed algorithm will be applied to a real inverter system in order to verify its application in the field.

\section{References}

[1] R. Naidoo, P. Pilay, "A New Method of Voltage Sag and Swell Detection," IEEE Trans. on Power Delivery, Vol. 22, No. 2, pp. 10561063 , Apr. 2007.

[2] N. Woodley, L. Morgan, A. Sundaram, "Experience with an inverter-based dynamic voltage restorer," IEEE Trans. on Power Delivery, Vol. 14, No. 3, pp. 11811186, July. 1999.

[3] W. Lee, D. Lee, T. Lee, "New Control Scheme for a Unified Power Quality Compensator-Q with Minimum Active Power Injection," IEEE Trans. on Power Delivery, Vol. 25, No. 2, pp. 10681076, Apr. 2010.
[4] W. Lee, W. Lee, T. Lee, "Integrated inverter system for compensation of Power Quality events," Power Electronics Specialists Conference, pp. 37543759, June. 2008,

[5] N. Tuaboylu, E. Collins, P. Chaney, "Voltage Disturbance Evaluation Using the Missing Voltage Technique", Harmonics and Quality of Power, Vol. 1, pp. 577582, 1998.

[6] M. Gonzalez, V.Cardenas, R. Alvarez, "A Fast Detection Algorithm for Sags, Swells, and Inter-ruptions Based on Digital RMS Calculation and Kalman Filtering," in Proc. Int. Power Electronics Congress, pp. 1-6, Oct. 2006

[7] Z. Yao, L Xiao, "Seamless transfer of single-phase grid-interactive inverters between grid-connected and stand-alone modes," IEEE Trans. on Power Electronics, Vol. 25, No. 6, pp. 1597-1603, 2010.

[8] Y. Silla, Y. Kum, "An Improvement in Synchronously Rotating Reference Frame-Based Voltage Sag Detection under Distorted Grid Voltages," Journal of Power Electronics, Vol. 8, No. 6, pp. 1283-1295, 2013.

[9] S. Lee, K. Sung, T. Lee, W. Lee "UPS/APF power conversion equipment with a seamless mode transfer," Energy Conversion Congress and Exposition (ECCE), pp. 2769 - 2776, May 2011.

[10] S. Lee, H. C, "Novel Fast Peak Detector for Singleor Three-phase Unsymmetrical Voltage Sags," Journal of Electrical Engineering \& Technology, Vol. 6, No. 5, pp. 658-665, Sep. 2011.

[11] J. S. Kim, Y. S. Kim, "Single-phase Active Power Filter Based on Rotating Reference Frame Method for Harmonics Compensation," Journal of Electrical Engineering \& Technology, Vol. 3, No. 1, pp. 94-100, Mar. 2008.

\section{Acknowledgements}

This work was supported by the GRRC program of Gyeonggi province. [(GRRC HANKYONG 2011-B04), Development of Power Conversion Equipment for Energy saving in Smart Distribution Center]

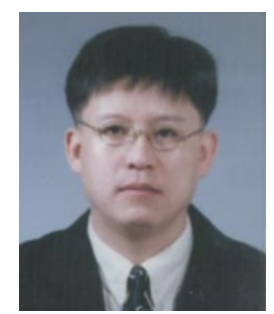

Woo-Cheol Lee He received the B.S. and M.S. and Ph.D. degrees in electrical engineering from Hanyang University, Seoul, Korea, in 1987, 1989, 2001, respectively. From 1988 to 1998 , he was with the R\&D Institute, Hyosung Industries Company Ltd., as a Senior Researcher, Seoul, Korea. He was a Visiting Professor in the department of Electrical Engineering at Virginia Polytechnic Institute and State University, Virginia, USA from 2007 to 2009. Since 2002, he has been with Hankyong National University, Ansung, 
Korea, where he is a Professor with the Department of Electrical, Electronic and Control Engineering. His research interests are in the areas of power converter, APF, UPS, and electrical drives.

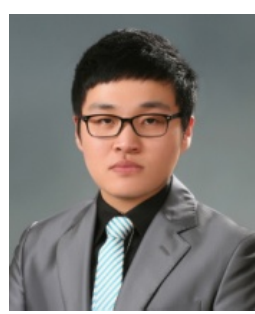

Kook-Nam Sung He received his B.S. and M.S. degrees from the Department of Electrical, Electronic and Control Engineering, Hankyong National University, Ansung, Korea. He currently working in the R\&D Institute, Green Power Company

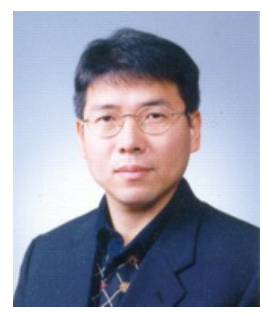

Taeck-Kie Lee received the B.S., M.S., and Ph.D. degrees in electrical engineering from Hanyang University, Seoul, Korea, in 1987, 1989, and 1993, respectively. From 1994 to 1996, he was with the Department of Electrical Engineering, Seonam University, Namwon, Korea. Since 1996, he has been with Hankyong National University, Ansung, Korea, where he is a Professor with the Department of Electrical Engineering. His research interests include power electronics, power converters, and electrical drives. 